\title{
Otimização da Formulação de uma Mistura Parte 1: Qualidade
}

\author{
Giovana Savitri Pasa
}

Mestranda do PPGEP / UFRGS

José Luis Duarte Ribeiro

Doutor em Engenharia - PPGEP / UFRGS

Danielle Barcos Nunes

Mestranda do PPGEP / UFRGS

Programa de Pós-Graduação em Engenharia de Produção - UFRGS

Praça Argentina, 10 9 - Porto Alegre, RS, 90040-020

Palavras-Chave: Qualidade, Otimização, Projeto de Experimentos, Formulações Químicas

Key words: Quality, Optimization, Design of Experiments, Chemical Mixture.

\section{RESUMO}

Este artigo descreve um trabalho de otimização da fomulação de uma mistura de borracha através do emprego de duas ferramentas principais: (i) Projeto de Experimentos com Misturas, usado no planejanento da coleta de dados; e (ii) Análise de Regressão Múltipla, usada na modelagem das características de qualidade.

O objet ivo deste trabalho é modelar o efeito dos fatores controláveis, ou seja, das quantidades dos ingredientes que compōem a mistura. sobre as caracteristicas de qualidade de interesse a deteminar quais os niveis ótimos destes fatores. ou seja. aqueles que conduzem as caracteristicas de qualidade ao melhor valor realizavel.

O traballıo foi realizado em um esforço conjunto empresa-universidade c consistiu das seguiultes etapas: Parte I - Identificação do processo em estudo, projeto e realização dos experimentos e ótimização da formulação com respeito à qualidade; Parte II - Otimização da fonmulação conciliando qualidade e custos. O presente artigo detém-se na descrição da primeira parte do trabalho, sendo a Parte II apresentada em um artigo subsec|üente.

\section{ABSTRACT}

This papes describes an optimization sfudy carred out on a mixture formulation. Two main tools uere used: (i) Design of Experiwents with Mixtures, used to help on collecting the data, and (ii) Multiple Regression. used to build models for the quality characteristics investigated.

The purpose of the study is to model the effects of the controllable factors (quantities of the components which constitute the mixture) on the quality characteristics, as well as to define the optimal set for these factors, i.e. the set which generates the hest possible values for the group of quality charcicteristics.

The work was accomplished as a company-university joint project and the following steps were performed: Part I - (i) process idenification: (ii) Design of an experiment; (iii) implementation of the experiment: and (iv) quality optimization of the mixture. Parlll - Mixthre optimization as a compromise of anality; and costs. 7 he present paper is limited to the discussion of Part I: in a forthcoming paper Part I/ will he presented.

Belo Horizonte, Vol. 6, No1, p.65-82 Jul. 1996 


\section{INTRODUÇÃO}

É prática comum no meio industrial realizar o desenvolvimento da formulação de misturas através de tentativa e erro, variando-se a proporção de uma matériaprima de cada vez no produto. Isso pode facilmente vir a consumir muito tempo, além de não possibilitar um entendimento das interações que possam existir entre os diversos componentes da mistura. A aplicação dos métodos de Projeto de Experimentos para misturas, por outro lado, requer menos tempo do que a prática de tentativa e erro, além de permitir uma otimização ou reformulação muito mais eficiente.

O uso do Projeto de Experimentos na otimização de formulações possui ampla aplicação em uma larga variedade de setores industriais, tais como os ramos alimentício, farmacêutico, químico e petroquímico (plásticos, tintas, combustíveis). Além da redução no tempo de desenvolvimento, a otimização experimental de misturas proporciona uma economia nos custos de Pesquisa \& Desenvolvimento de produtos, podendo ainda trazer diminuições no custo de matérias-primas e simplificações no processo. Constitui-se, dessa forma, em importante vantagem competitiva, conforme enfatizado por Heinsman \& Montgomery (1995).

No planejamento de experimentos, em geral, o espaço experimental é restrito somente pelas faixas de variação viáveis para cada fator controlável, não havendo dependência entre os níveis adotados para os mesmos (Montgomery, 1984). Em experimentos com misturas, entretanto, é preciso respeitar o fato de que as proporções dos ingredientes devem ter sua soma igual a 1 e de que nenhum ingrediente pode assumir valores negativos.

Ou seja, para uma mistura contendo q ingredientes, $x_{i}$ (a proporção do i-ésimo componente) deve respeitar a restrição

$0 \leq x_{i} \leq 1 \quad$ com $\quad i=1,2, \ldots, q$

e, para cada rodada do experimento,

$$
x_{1}+x_{2}+\ldots+x_{q}=1
$$

Ilustrando, para uma mistura de $q=3$ componentes, a Eq. (2) será $x 1+x 2+x 3=1$, e o espaço experimental da mistura estará restrito ao triângulo equilátero sombreado mostrado na Figura 1. Se a mistura em questão fosse composta por quatro componentes, o espaço experimental seria um tetraedro regular. Os vértices da região viável representam misturas puras (um só componente), as arestas são misturas binárias, e o interior da região são misturas completas (contendo todos os ingredientes).

Em um projeto de experimentos com fatores independentes, todos os vértices do cubo (além de quaisquer pontos nas arestas ou no interior do mesmo) são pontos experimentais viáveis. No entanto, o espaço viável para um experimento com mistura estará sempre contido em uma superficie limitada pelas Eqs. (1) e (2). 
A essas restrições, é comum sobreporemse outras, relacionadas às faixas de variação possiveis para as proporções de cada ingrediente, tais como:

$$
\text { LIi } \leq x i \leq \text { LSi , } i=1,2, \ldots, \text { q. (3) }
$$

Esses limites estabelecem que cada mistura deve conter uma proporção mínima (Lii) do ingrediente $i$, e não mais do que uma proporção máxima (LSi) do mesmo. O efeito dessas restrições é reduzir ainda mais o tamanho e, provavelmente, alterar a forma da região viável de experimentação. Por exemplo, se limites superior e inferior forem impostos ao espaço de experimentos da mistura de três componentes representado na Figura 1, a região viável passará a ser um poliedro irregular, e não mais o triângulo equilátero mostrado. Diversos outros exemplos podem ser encontrados em Cornell (1973).

Dessa forma, experimentos com misturas sempre requerem técnicas especiais de planejamento, pois cada rodada ou combinação de tratamentos deve ser uma mistura viável, isto é, deve estar dentro da região limitada por todas as restrições de limites inferiores e superiores.

O objeto de estudo neste trabalho é um processo de produção de borracha. $\mathrm{Na}$ receita da borracha há vários ingredientes, e as características de qualidade do produto final (borracha) são funções somente das proporções destes ingredientes e não das quantidades absolutas dos mesmos. Por isso, o processo eml questão enquadra-se como um problema de misturas, conforme definido anteriormente.

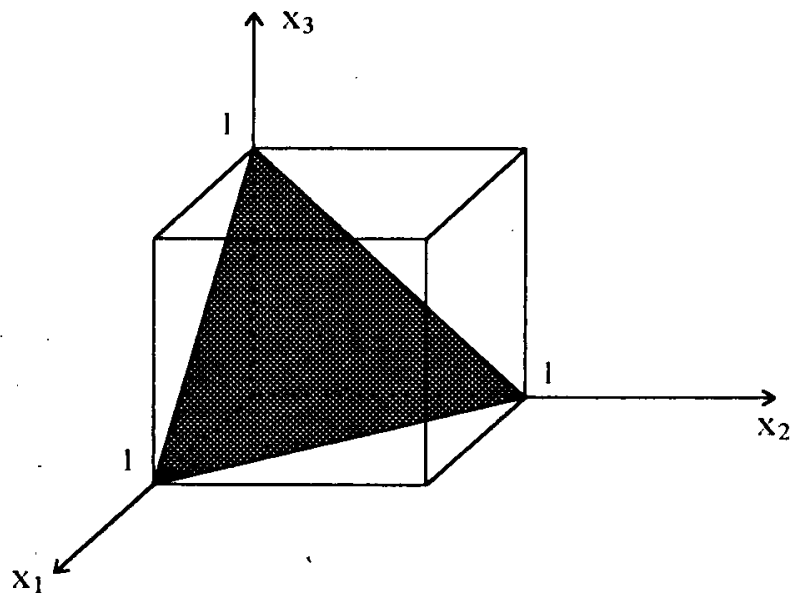

Figura 1: Representação cartesiana do espaço experimental de uma mistura (com a proporção de cada fator controlável podendo variar de 0 a 1 ) 


\section{APRESENTAÇĀO DO ESTUDO DE CASO}

O trabalho de otimização da qualidade de uma mistura de borracha dividiu-se nas seguintes elapas:

- Identificação dos fatores controláveis e caracteristicas de qualidade de interesse:

Os fatores controláveis $\mathrm{e}$ as características de qualidade a serem considerados no estudo de otimização experimental foram escolhidos de acordo com o conhecimento dos engenheiros responsáveis pelo processo.

A mistura de borracha em questão é composta por vários ingredientes, dos quais. quatro foram escolhidos como fatores controláveis. Para manter os dados confidenciais, esses ingredientes serão designados por $\mathrm{X} 1, \mathrm{X} 2, \mathrm{X} 3$ e X4. Os demais ingredientes que compõem a mistura foram mantidos constantes durante todo o estudo. Na Tabela I, são apresentadas as faixas de variação percentual aceitas para cada um dos componentes. A partir das faixas de variação, tem-se o espaço experimental possível dentro do qual foram planejados os ensaios.

As características de qualidade (CQ) consideradas na otimização da formulação foram seis: Abrasão, Densidade, Durezal, Tensão de Ruptura, Módulo 300\% e Alongamento. As mesmas serão daqui por diante referidas como $Y_{1}, Y_{2}, \ldots, Y_{0}$ (não necessariamentc na mesmà ordem em que foram citadas, para preservar a confidencialidade dos dados).

\begin{tabular}{|c|c|c|}
\hline & \multicolumn{2}{|c|}{ Faixa de Variação (em \%) } \\
\hline $\begin{array}{c}\text { Fator } \\
\text { controlável }\end{array}$ & Mínimo & Má x i mo \\
\hline X 1 & 0,673 & 54,60 \\
\hline X2 & 10,01 & 50,00 \\
\hline X3 & 33,31 & 44,00 \\
\hline X4 & 0,000 & 7,335 \\
\hline
\end{tabular}

Tabela 1 - Faixas de variação possível dos fatores controláveis 


\section{PRODUÇÃO}

Conforme os respectivos valores alvo e limites de especificação, pôde-se classificar cada CQ como do tipo "maioré-melhor" (quando possui apenas limite inferior de especificação), "menor-émelhor" (apenas limite superior de especificação) ou "nominal-é-melhor" (ambos) (Ver Anexo 1). Além disso, de acordo com a experiência dos engenheiros responsáveis, a cada CQ foi atribuído um peso, correspondente à sua importância relativa na avaliação da qualidade global da mistura. Esses valores são apresentados na.Tabela 2 a seguir.

- Planejamento do Experimento e Coleta de Dados:

Foram realizados de acordo com as técnicas de Projeto de Experimentos com Misturas e serão descritos a seguir.
- Análise dos Resultados Experimentais:

Os dados coletados foram analisados com o auxílio de Análise de Regressão Múltipla (ver por exemplo Montgomery \& Peck, 1991), obtendo-se modelos capazes de estimarem o efeito dos fatores controláveis sobre cada uma das CQ de interesse.

- Otimização da Qualidade da Formulação:

A partir dos modelos ajustados por regressão, determinaram-se quais os niveis ótimos dos fatores controláveis para cada uma das $C Q$ individuais (aqueles que fazem com que a $\mathrm{CQ}$ em questão assuma o melhor valor possível dentro das especificações do cliente).

\begin{tabular}{cccccc}
\hline CQ & Importância & Tipo & Alvo & Mínimo & \multicolumn{2}{c}{$\begin{array}{c}\text { Limites de } \\
\text { Especificação }\end{array}$} \\
Y1 & 2,25 & menor-é-melhor & 111 & - & 113 \\
Y2 & 1,75 & nominal-é-melhor & 68 & 63 & 70 \\
Y3 & 1,50 & maior-é-melhor & 320 & 270 & - \\
Y4 & 1,00 & maior-é-melhor & 110 & 90 & - \\
Y5 & 1,25 & maior-é-melhor & 110 & 90 & 230 \\
Y6 & 2,00 & menor-é-melhor & 180 & - & \\
\hline
\end{tabular}

Tabela 2 - Características de Qualidade (CQ) 
A seguir, determinou-se qual o ajuste ótimo global dos fatores controláveis, considerando-se todas as características de qualidade simultaneamente, ponderadas por seus respectivos pesos. Para isto, fezse uso de uma Função de Perda proposta por Ribeiro e Elsayed (1995).

\section{PLANEJAMENTO DO EXPERIMENTO}

Para o planejamento da coleta de dados, utilizou-se a técnica de Projeto de Experimentos com Misturas. Conforme mencionado, no processo em questão foram considerados quatro ingredientes: $X_{1}$ , $X_{2}, X_{3}$ e $X_{4}$. Assim, os ensaios foram planejados de forma que a soma das proporções em massa de cada um dos quatro componentes da mistura sempre resultasse em $100 \%$.

Para realizar o planejamento do experimento, foi utilizado um sistema de eixos (Figura 2), o qual nada mais é do que uma representação cartesiana (rebatimento) dos eixos $x_{1}, x_{2}$ e $x_{3}$ que aparecell na Figura 1.

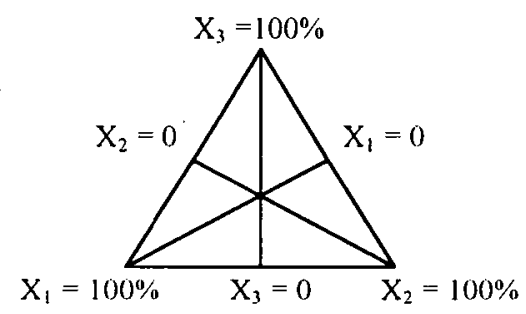

Figura 2: Sistema de cixos utilizado no planejamento
Inicialmente, representou-se o espaço experimental delimitado pelas restrições de três dos componentes. No espaço experimental viável, para diversos níveis do quarto componente $\left(X_{4}\right)$, foram distribuidos pontos (Figura 3) respeitando-se os seguintes critérios de distribuição:

(i) distribuição aproximadamente uniforme de pontos sobre o espaço experimental viável correspondente a cada nível de $X_{4}$,

(ii) número de níveis suficiente para pesquisar efeitos não lineares (quadráticos).

A Tabela 3, abaixo, especifica os limites de experimentação dos fatores controláveis $X_{1}$ e $X_{2}$ para cada nível de $\mathrm{X}_{4}$ utilizado no planejamento. Esses limites foram definidos, levando-se em conta caracteristicas especificas do processo, que impõem uma relação entre as quantidades em massa dos componentes $X_{1}$ e $X_{2}$. Para todos os niveis de $X_{4}$, o componente $X_{3}$ pode variar de 33,31 a $44,00 \%$, como definido anteriormente.

Construiu-se, então, a matriz experimental, apresentada na Tabela 4 (os níveis dos fatores controláveis estão expressos em quantidades percentuais). 


\begin{tabular}{c|cccc}
\hline $\begin{array}{c}\text { Nível do } \\
\text { componente }\end{array}$ & Faixa de Variação de X1 & Faixa de Variação de X2 \\
\cline { 2 - 5 } & Mínimo & Máximo & Mínimo & Máximo \\
\hline 0,0000 & 28,01 & 54,60 & 10,01 & 30,01 \\
2,000 & 24,01 & 43,97 & 15,37 & 35,33 \\
3,674 & 17,68 & 37,65 & 20,02 & 39,99 \\
5,350 & 11,35 & 31,33 & 24,66 & 44,64 \\
7,335 & 0,673 & 27,34 & 30,01 & 50,00
\end{tabular}

Tabela 3 - Faixas de variação possível para $X_{1}$ e $X_{2}$ a cada nível de $X_{4}$

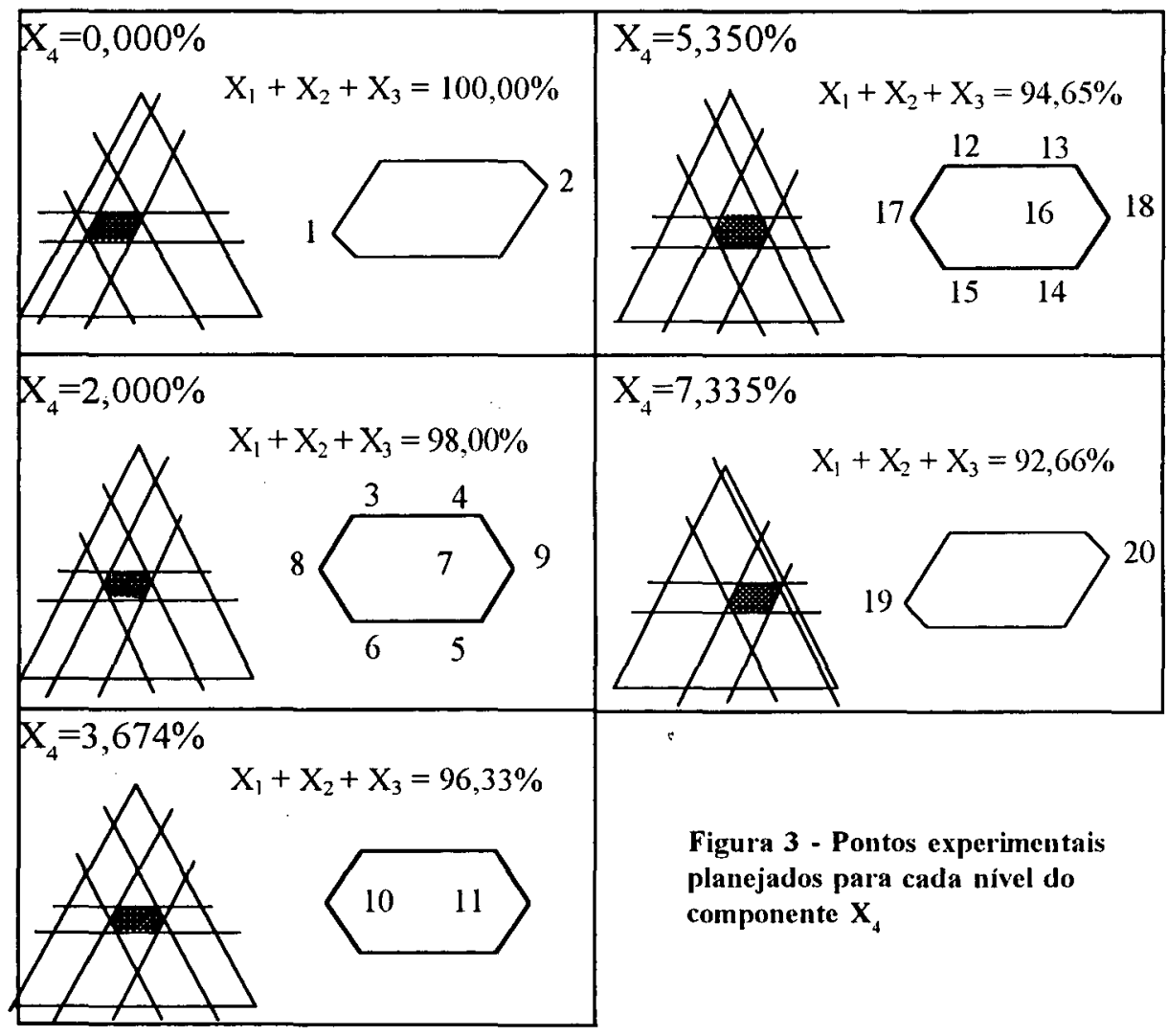




\section{PRODUÇÃO}

\begin{tabular}{|c|c|c|c|c|c|}
\hline Ensaio & $\times 1$ & $\times 2$ & $\times 3$ & $\times 4$ & TOTA L \\
\hline 1 & 54.60 & 10,01 & 35,34 & 0,000 & 100,00 \\
\hline 2 & 28,01 & 30,01 & 41,98 & 0,000 & 100,00 \\
\hline 3 & 35,98 & 18,02 & 44,00 & 2,000 & 100,00 \\
\hline 4 & 26,66 & 27,35 & 44,00 & 1,994 & 100,00 \\
\hline 5 & 31,99 & 32,67 & 33,33 & 2,013 & 100,00 \\
\hline 6 & 41,35 & 23,35 & 33,31 & 1,993 & 100,00 \\
\hline 7 & 33,98 & 25,37 & 38,65 & 2,003 & 100,00 \\
\hline 8 & 43,97 & 15,33 & 38,68 & 2,019 & 100,00 \\
\hline 9 & 24,01 & 35,36 & 38,66 & 1,972 & 100,00 \\
\hline 10 & 27,66 & 29,99 & 38,68 & 3,674 & 100,00 \\
\hline 11 & 27,66 & 29,99 & 38,68 & 3,674 & 100,00 \\
\hline 12 & 23,34 & 27,34 & 44,00 & 5,316 & 100,00 \\
\hline 13 & 14,00 & 36,69 & 44,00 & 5,312 & 100,00 \\
\hline 14 & 19,34 & 41,97 & 33,31 & 5,377 & 100,00 \\
\hline 15 & 28,67 & 32,67 & 33,31 & 5.350 & 100,00 \\
\hline 16 & 21,34 & 34,65 & 38,66 & 5,351 & 100,00 \\
\hline 17 & 31,33 & 24,68 & 38,67 & 5.316 & 100,00 \\
\hline 18 & 11,35 & 44,64 & 38,65 & 5,360 & 100,00 \\
\hline 19 & 27,34 & 30,01 & 35,33 & 7,319 & 100,00 \\
\hline 20 & 0,673 & 50,00 & 41,99 & 7,335 & 100,00 \\
\hline
\end{tabular}

Tabela 4 - Matriz experimental para a coleta de dados

\section{TRANSFORMAÇÃO DE VARIÁVEIS}

Após a coleta de dados experimentais, o passo que precede a otimização é a transformação das quatro variáveis $X_{i}$ (fatores controláveis). Uma vez que as mesmas apresentam uma relação de dependência linear $\left(X_{1}+X_{2}+X_{3}+X_{4}=\right.$ 1 , ou $100 \%$ ), devem ser transformadas em três variáveis independentes, as quais serão denominadas $\mathrm{W}_{1}, \mathrm{~W}_{2} \mathrm{e} \mathrm{W}_{3}$

A necessidade de se realizar tal transformação deve-se à maior precisão matemática obtida na determinação das regiões de ajustes ótimos realizáveis (misturas) ao se trabalhar com variáveis independentes. O procedimento de transformação execulado foi proposto por Cornell (1981) e será descrito a seguir. 
O primeiro passo da transformação é a passagem dos niveis dos fatores controláveis $\left(\mathrm{X}_{1}, \mathrm{X}_{2}, \mathrm{X}_{3}\right.$ e $\left.\mathrm{X}_{4}\right)$ expressos em percentuais (Tabela 4) para variáveis intermediárias $\left(V_{1}, V_{2}, V_{3}\right.$ e $\left.V_{4}\right)$. A transformaç̃̃o para variáveis intermediárias é feita através da aplicação da equação apresentada a seguir.

$$
V_{i}=\frac{X_{i}-X_{o i}}{h_{i}} \mathrm{i}=1,2, \ldots, \mathrm{q}
$$

onde

$V_{i}$ é a variável intermediária i,

$X_{i}$ é o fator controlável i expresso em percentual,

$X_{o i}$ é a média da faica de variação do fator controlável i (percentual),

$h_{j}$ é a semi-amplitude da faixa de variação do fator controlável i (percentual),

q é o número de componentes da mistura.

A transformação de $X_{i}$ para $V_{i}$ é tal que $\sum_{i=1}^{q} V_{i}^{2} \leq 1$. Essa transformação de variáveis é realizada para cada rodada do experimento, e a região experimental passa a ser definida como um elipsóide q-dimensional.

O passo seguinte é transformar as variáveis intermediárias $\mathrm{V}_{\mathrm{i}}$, as quais ainda possuem uma relação linear, em variáveis independentes $W_{i}$. Como há uma relação linear entre as quantidades dos componentes de uma mistura (Eq. 2), uma vez determinadas as quantidades de três dos componentes, está determinada a quantidade do quarto. Então, na realidade, pode-se reduzir a matriz experimental de quatro componentes dependentes para uma matriz experimental com três componentes independentes. Para isto, deve-se encontrar uma matriz ortogonal $\mathrm{T}$ de ordem $\mathrm{q} \times \mathrm{q}$, tal que

$$
V T=[\mathbf{W} \mid \mathbf{0}]
$$

\begin{tabular}{|c|c|c|}
\hline $\begin{array}{c}\text { Fator } \\
\text { Controlável }\end{array}$ & $\begin{array}{c}\text { Média } \\
\text { Xoi }\end{array}$ & $\begin{array}{c}\text { Semi-amplitude } \\
\text { hi }\end{array}$ \\
\hline X1 & 27,66 & 26,98 \\
\hline X2 & 30,01 & 19,99 \\
\hline X3 & 38,66 & 5,348 \\
\hline$X 4$ & 3,668 & 3,668 \\
\hline
\end{tabular}

Tabela 5 - Médias e semi-amplitudes das faixas de variação dos $X_{\text {, }}$ 


\section{PRODUÇÃO}

onde

$V$ é a matriz de variáveis intermediárias de ordem $\mathrm{N} \times \mathrm{q}$

(N é o número de ensaios),

T é uma matriz ortogonal de ordem q $x q$,

W é a matriz de variáveis independentes de ordem $\mathrm{N} \times(\mathrm{q}-\mathrm{l})$,

0 é o vetór $\mathrm{N} \times 1$ de zeros.

A matriz $\mathbf{T}$ ortogonal foi determinada a partir dos procedimentos sugeridos por Cornell (1981, p. 108). Essa matriz é da forma $\mathbf{T}=\left[\mathbf{T}_{1} \mid \mathbf{T}_{2}\right]$, onde $\mathbf{T}_{1}$ é matriz de ordem $q \times(q-1)$, e $T_{2}$ é matriz coluna de ordem $q$, de modo que V.T $\mathbf{T}_{1}=\mathbf{W}$ e $\mathbf{V} \cdot \mathbf{T}_{2}=\mathbf{0}$.

Observando-se o fato de que

$$
\sum_{i=1}^{q} X_{i}=\sum_{i=1}^{q} X_{o i}=1
$$

e, a partir da equação (4),

$$
\sum_{i=1}^{q}\left(X_{i}-X_{o i}\right)=\sum_{i=1}^{q} h_{i} V_{i},
$$

obtém-se a relação

$$
\sum_{i=1}^{q} h_{i} V_{i}=0
$$

através da qual se pode, sem perda de generalidade, determinar a matriz $\mathbf{T}_{2}$.
Faz-se, então, $\widetilde{h}_{i}=\frac{h_{i}}{\left(\sum_{i=1}^{q} h_{i}^{2}\right)^{1 / 2}}$,

mantendo-se $\sum_{i=1}^{q} \widetilde{h}_{i} V_{i}=0$.

Assim, o vetor $\mathbf{T}_{2}$ pode ser definido simplesmente como

$\mathbf{T}_{2}^{\prime}=\left[\begin{array}{llll}\tilde{h}_{1} & \tilde{h}_{2} & \tilde{h}_{3} & \tilde{h}_{4}\end{array}\right]$

Os elementos da matriz $T_{1}$ também podem ser obtidos usando-se os $h_{i}$ 's $(\mathrm{l} \leq \mathrm{i} \leq \mathrm{q})$ :

$$
\begin{array}{ll}
t_{11}=-h_{2} & t_{12}=-h_{1} h_{3} \\
t_{21}=h_{1} & t_{22}=-h_{2} h_{3} \\
t_{31}=0 & t_{32}=h_{1}^{2}+h_{2}^{2} \\
t_{41}=0 & t_{42}=0
\end{array}
$$

$$
\begin{aligned}
& t_{13}=-h_{1} h_{4} \\
& t_{23}=-h_{2} h_{4} \\
& t_{33}=-h_{3} h_{4} \\
& t_{43}=h_{1}^{2}+h_{2}^{2}+h_{3}^{2}
\end{aligned}
$$

e então as colunas acima são notmalizadas, gerando-se a matriz $\mathbf{T}_{\mathbf{1}}$. 
Dessa forma, pode-se obter a matriz $\mathbf{W}=\mathbf{V} . \mathbf{T}_{1}$, cujos valores resultantes são apresentados na Tabela 6.

\begin{tabular}{cccc}
\hline Ensaio & W1 & W2 & W3 \\
\hline 1 & $-1,399$ & $-0,6441$ & $-1,013$ \\
2 & $-0,0071$ & 0,6107 & $-1,013$ \\
3 & $-0,6653$ & 1,003 & $-0,4602$ \\
4 & $-0,085$ & 1,003 & $-0,4622$ \\
\hline 5 & 0,01097 & $-1,016$ & $-0,4572$ \\
6 & $-0,5701$ & $-1,021$ & $-0,4633$ \\
7 & $-0,3258$ & $-0,0087$ & $-0,4602$ \\
8 & $-0,9501$ & $-0,0042$ & $-0,4561$ \\
\hline 9 & 0,2949 & $-0,0069$ & $-0,4683$ \\
10 & $-0,0004$ & 0,0039 & 0,00175 \\
11 & $-0,0004$ & 0,0039 & 0,00175 \\
\hline 12 & $-0,0116$ & 1,02 & 0,455 \\
13 & 0,5697 & 1,019 & 0,455 \\
14 & 0,6639 & $-1,005$ & 0,4723 \\
15 & 0,0845 & $-1,005$ & 0,4652 \\
\hline 16 & 0,3258 & 0,00888 & 0,4652 \\
17 & $-0,2947$ & 0,0104 & 0,4561 \\
18 & 0,9479 & 0,00581 & 0,4672 \\
19 & 0,00691 & $-0,6129$ & 1,009 \\
20 & 1,399 & 0,649 & 1,013 \\
\hline & & & \\
\hline
\end{tabular}

Tabela 6 - Matriz experimental em função das variáveis independentes $W_{i}$
A análise da Tabela 6 indica que as variáveis $W$ mantêm as seguintes relações com as variáveis $V_{i}$ e $\mathbf{X}_{i}$ :

$W_{1}$ é uma combinação linear de $V_{1}$ e $\mathrm{V}_{2}$, ou $\left(\mathrm{X}_{1}-\mathrm{X}_{\mathrm{ol})}\right.$ e $\left(\mathrm{X}_{2}-\mathrm{X}_{\mathrm{o} 2}\right)$

$\mathrm{W}_{2}$ é proporcional a $\mathrm{V}_{3}$, ou $\left(\mathrm{X}_{3}-\mathrm{X}_{\mathrm{e} 3}\right)$

$\mathrm{W}_{3}$ é proporcional a $\mathrm{V}_{4}$, ou $\left(\mathrm{X}_{4}-\mathrm{X}_{\mathrm{o4}}\right)$

\section{OTIMIZAÇÃO}

Através da análise dos dados coletados, determinaram-se modelos que relacionam o valor das Características de Qualidade de interesse com a quantidade de cada componente na mistura. A partir desses modelos individuais, foram obtidos os ajustes dos fatores controláveis (proporções dos componentes) capazes de conduzir à formulação que apresenta a melhor qualidade possível.

\subsection{Modelos e Otimização para as} Caracteristicas de Qualidade Individuais

Os modelos para cada uma das Caracteristicas de Qualidade de interesse foram obtidos através de Regressão Múltipla, em função das variáveis independentes $W_{1}, W_{2}$ e $W_{3}$. Após, determinaram-se os ajustes ótimos individuais de cada Característica de Qualidade (CQ), ou seja, aqueles ajustes que permitem que a $\mathrm{CQ}$ em questão assuma o melhor valor possivel. 
$Y_{1}=113,6-0,5651 \times W_{1}+2.972 \times W_{2}+0,2945 \times W_{3}+0,212 \times W_{2}^{2} \quad s_{1}=0,1236$

$Y_{2}=64.9+2.109 \times W_{1}+5,881 \times W_{2}-1,511 \times W_{3}$

$S_{2}=0.7529$

$\mathrm{Y}_{3}=315,4-8,458 \times \mathrm{W}_{1}+8,75 \times \mathrm{W}_{3}+23,78 \times \mathrm{W}_{3}^{2}$

$Y_{4}=159,65+24,66 \times W_{1}+67,51 \times W_{2}-14,94 \times W_{3}+$

$+9,979 \times W_{1}^{2}+14,06 \times W_{2}^{2}$

$Y_{5}=93,3-11,03 \times W_{1}-17,84 \times W_{2}+$

$+6,668 \times W_{3}-3,642 \times W_{1}{ }^{2}+5,572 \times W_{3}^{2}$

$Y_{6}=182,2-14,176 \times W_{1}+11,252 \times W_{3}-4,990 \times W_{1} \times W_{3}+$

$+8.228 \times \mathrm{W}_{2}^{2}+4.256 \times \mathrm{W}_{3}^{2}$

Tabela 7: Modelos para a Estimativa das Características de Qualidade em Funçāo dos W,

\begin{tabular}{|c|c|c|c|c|c|c|c|c|c|c|}
\hline & \multicolumn{3}{|c|}{ AJUSTES ÓTIMOS } & \multicolumn{6}{|c|}{ RESPOSTAS } & \multirow[b]{2}{*}{ PERDA } \\
\hline CQ & W1 & $\mathrm{W} 2$ & W3 & Y1 & Y2 & $\mathrm{Y} 3$ & Y4 & Y5 & Y6 & \\
\hline Y1 & 1 & -1 & -1 & 110,0 & 62.64 & 322.0 & 155.8 & 95.38 & 174.24 & 3.06 \\
\hline Y2 & 0.31 & 0.33 & -0.33 & 114.3 & 68.00 & 312.5 & 197.0 & 82.00 & 176.00 & 9.10 \\
\hline Y3 & -1 & -1 & 1 & 111.7 & 55.40 & 356,4 & 76.58 & 130.8 & 225.2 & 16.2 \\
\hline$Y_{4}$ & 0.51 & 0,51 & -0.51 & 114.7 & 69,75 & 312.8 & 220.5 & 75,68 & 173.78 & 13,47 \\
\hline YS & -1 & -1 & 1 & 111.7 & 55,40 & 356.4 & 76.58 & 130,8 & 225.2 & 16,2 \\
\hline$Y_{6}$ & 1 & 0 & -0.74 & 112.8 & 68.13 & 313.5 & 205,3 & 76,74 & 165,72 & 5,93 \\
\hline
\end{tabular}

Tabela 8: Ajustes ótimos para as Caracteristicas de Qualidade Individuais 


\subsection{OTIMIZAÇÃO DA QUALIDADE - ATRAVÉS DA FUNÇÃO DE PERDA}

Para determinar-se qual o ajuste ótimo global, é necessário fazer uso de uma expressão matemática capaz de avaliar simultaneamente todas as Caracteristicas de Qualidade de interesse, ponderadas pelas suas respeclivas importâncias. Uma expressão capaz de fazer isto é a Função de Perda proposta por Ribeiro e Elsayed (1995), cujos exemplos de aplicação podem ser encontrados em Caten e Ribeiro (1995) e Caten e Ribeiro (1995b). Essa função é composta por três parcelas, descritas a seguir.

A primeira parcela diz respeito à perda decorrente da diferença entre o valor médio especificado para uma dada característica de qualidade do produto e o valor médio efetivamente obtido pelo processo produtivo. Considera-se a perda diretamente proporcional ao quadrado destal diferença.

A segunda parcela diz respeito à variabilidade da caracteristica de qualidade, observada quando se avaliam várias unidades do produto geradas com um mesmo ajuste do processo. A perda é considerada diretamente proporcional à variância dos valores obtidos para a característica de saida do produto.

A terceira parcela diz respeito à sensibilidade das características de qualidade do produto à imprecisão dos ajustes nos fatores controláveis. Considera-se que a perda é diretamente proporcional ao produto da variabilidade de um certo parâmetro do processo pela sensibilidade da característica de qualidade a esta variabilidade.

Equação da Função de Perda (genérica),

$\hat{\bar{u}}(k)=\sum_{j=1}^{J}\left[w_{j}\left[(D M)^{2}+\hat{\sigma}_{Y_{j}}{ }^{2}+\sum_{i=1}^{l}\left(\hat{\sigma}_{X_{i}}{ }^{2}\left(\frac{{\hat{\alpha_{j}}}^{2}}{\partial_{X_{i}}}\right)^{2}\right)\right]\right]$

onde: $\hat{Z}(k)$ é o estimador para a perda " $Z$ ", em função do ajuste " $k$ " dos fatores controláveis;

$w_{j}$ é um fator multiplicativo que tem duas funções: normalizar os valores que representam os desvios do alvo, obtidos nas unidades de grandeza da caracteristica de qualidade, para que os desvios das seis CQ possam ser diretamente comparáveis; e atribuir a cada CQ um peso correspondente à sua importância relativa, de forma que as parcelas da função perda correspondentes a uma dada CQ fiquem ponderadas pela sua importância;

DM é o desvio do alvo, que faz com que o cliente experimente uma perda de qualidade. O Anexo 1 contém o formulário para o cálculo de DM, o qual depende do tipo da caracteristica de 


\section{PRODUÇÃO}

qualidade (maior-é-melhor, nominal-émelhor, etc.)

$\hat{Y}_{j}$ é o modelo matemático que fornece uma estimativa da média da CQ "j" em função do ajuste dos fatores controláveis;

$$
\hat{\sigma}_{Y_{j}} \text { é o modelo matemático que }
$$
fornece uma estimativa do desvio padrão da CQ " $\mathrm{j}$ " em função do ajuste dos fatores controláveis;

$X_{i}$ é o parâmetro de processo " $\mathrm{i}$ ";

$\hat{\sigma}_{X_{i}}$ é a estimativa do desvio padrão (variabilidade) do parâmetro de processo "i".

A perda global será então avaliada através da aplicação da Eq. 11 ao processo em estudo. O próximo passo é calcular os coeficientes $w_{j}$, que são dados pela seguinte expressão:

$w_{j}=F \times$ importância relativa da CQ

onde $\mathrm{F}$ é um fator que depende do tipo da Característica de Qualidade:

- Maior-é-melhor: $F=1 /$ alvo mínimo ${ }^{2}$

- Menor-é-melhor: $F=1 /($ máximo alvo) ${ }^{2}$

- Nominal-é-melhor, com perda simétrica para desvios acima ou abaixo do alvo:

$\mathrm{F}=4 /(\text { máximo - mínimo })^{2}$

- Nominal-é-melhor, com perda assimétrica em relação ao alvo:

$\mathrm{F}=1 /(\text { máximo }- \text { alvo })^{2}$ se $Y_{j}>T_{j}$
$F=1 /(\text { alvo }- \text { mínimo })^{2}$ se $Y_{j}<T_{j}$

Para a terceira parcela da função de perda, é necessário informar qual a variabilidade dos fatores controláveis e qual a sensibilidade das caracteristicas de qualidade a esta variabilidade. No caso em estudo, observou-se que a variabilidade dos fatores controláveis poderia ser desprezada; dessa forma, não é necessário estimar a sensibilidade.

\section{Otimização global:}

A Função de Perda encontrada para o processo em questão é constituida de muitos termos, cada um deles responsável por representar somente uma pequena parte do modelo.

Através desta Função, simula-se qual seria a perda para um certo conjunto de ajustes dos fatores controláveis. A partir destes pontos simulados, realiza-se uma nova Regressão Múltipla e obtém-se um novo modelo.

Este novo modelo traz consigo todos os benefícios de ser um modelo mais enxuto e mantém a representatividade do modelo original. Ele é denominado metamodelo e é apresentado a seguir, expresso em termos das variáveis independentes $\mathrm{W}$.

Metamodelo obtido para a Perda Global:

Perda $=5,634-1,1 W_{1}+8,043 W_{2}+$ $0,8536 \mathrm{~W}_{3}+2,493 \mathrm{~W}_{1} \cdot \mathrm{W}_{2}-1,804 \mathrm{~W}_{1} \cdot \mathrm{W}_{3}$ $-1,823 \mathrm{~W}_{2} \cdot \mathrm{W}_{3}+1,513 \mathrm{~W}_{1}{ }^{2}+10,87 \mathrm{~W}_{2}{ }^{2}+$ $2,31 \mathrm{~W}_{2}{ }^{3}$ 
Usando o modelo anterior, o ajuste ótimo global resulta:

\begin{tabular}{|c|c|c|c|c|c|c|c|c|c|}
\hline W & W & WB & Y & Y & Y3 & Y4 & Y & Y6 & Pada \\
\hline 0,53 & 0,55 & 1 & 111,4 & 64,3 & 325,9 & 157,6 & 95,2 & 172,8 & 2,14 \\
\hline
\end{tabular}

Expresso em percentual:

\begin{tabular}{|c|c|c|c|c|}
\hline $\mathrm{X} 1$ & $\mathrm{X} 2$ & $\mathrm{X} 3$ & $\mathrm{X} 4$ & $*$ total \\
\hline 23,31 & 40,82 & 35,85 & 0,00 & 100,00 \\
\hline
\end{tabular}

Observa-se que este ajuste prevê $\mathrm{X}_{4}$ de de preparo, simplificação das operações, $0,00 \%$, ou seja, trata-se de uma mistura que dispensa o uso desse componente. Em geral, a eliminação de um componente simplificação do estoque, etc.

A análise dos gráficos de sensibilidade traz vantagens econômicas: menor tempo e das curvas de isovalores para a perda

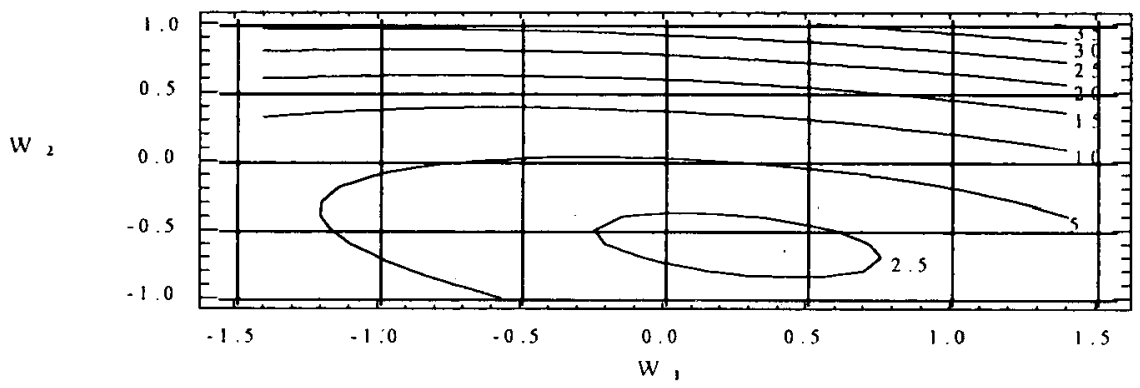

Figura 4: Curvas de isovalores da Perda Global, traçadas para $W_{3}=-1$ 


\section{PRODUÇÃO}

global permite identificar uma região operacional ótima para as quantidades dos ingredientes. Essa região seria a seguinte:

\begin{tabular}{|c|c|c|c|}
\hline W & ve & w & Puxh \\
\hline $0,0 \mathrm{a} 0,6$ & $0,7 \mathrm{a}-0,4$ & $-1,0 \mathrm{a} 0,8$ & $<30$ \\
\hline
\end{tabular}

Expresso em percentual:

\begin{tabular}{|c|c|c|c|c|}
\hline $\mathrm{X}$ & $\mathrm{X}$ & $\mathrm{x}$ & $\mathrm{X}$ & Perda \\
\hline $22 \mathrm{a} 32$ & $34 \mathrm{a} 46$ & $\begin{array}{c}34,1 \mathrm{a} \\
36,8\end{array}$ & $0 \mathrm{a} 1,0$ & $<30$ \\
\hline
\end{tabular}

\section{CONCLUSÕES}

Este trabalho descreve um estudo de otimização experimental da formulação de uma mistura de borracha. Inicialmente, foi planejado um experimento envolvendo os fatores controláveis (ingredientes da mistura) de interesse. O planejamento foi feito usando-se técnicas especificas para o Projeto de Experimentos de misturas.

Após, foi feila a coleta de dados que contemplou um total de 20 tratamentos (20 diferentes formulações). Para cada tratamento, foi medido e registrado o valor das seguintes características de qualidade: Abrasão, Densidade, Dureza, Tensão de ruptura, Módulo 300 e Alongamento.

Os resultados obtidos foram analisados usando-se as técnicas de regressão múltipla. Com o auxílio dos modelos de regressão, foi possível identificar os ajustes ótimos (proporções ótimas dos ingredientes) considerando, isoladamente, cada característica de qualidade.

Por fim, determinou-se o ajuste ótimo da qualidade da mistura, considerandose simultaneamente todas as características de qualidade. A busca da qualidade ótima foi feita usando-se uma função de perda multivariada proposta por Ribeiro e Elsayed (1995).

Em artigo subseqüente, será descrita a Parte II deste trabalho, que determinou um ajuste ótimo global, conciliando qualidade e custos de produção.

O trabalho se constituiu em um projeto conjunto empresa-universidade e ambas as partes foram beneficiadas. É importante destacar as vantagens que esse trabalho pôde proporcionar para a empresa:

- Expôs o corpo técnico da empresa ao estudo e aplicação de técnicas específicas para o planejamento de experimentos $\mathrm{c}$ otimização de misturas;

- Catalisou a definição formal dos valores alvos e especificações para cada uma das característica de qualidade do produto estudado; 


\section{PRODUÇÃO}

- Contém informações precisas a respeito do efeito dos ingredientes investigados sobre cada uma das características de qualidade;

- Traz a indicação do ajuste ótimo, que pode conduzir a uma importante melhoria da qualidade total do produto.

ANEXO 1

\begin{tabular}{|c|c|c|}
\hline $\begin{array}{c}\text { Tipo da Caracteristica } \\
\text { de Qualidade }\end{array}$ & $\begin{array}{c}\text { REPRESENTAÇĀO GRÁFICA DA } \\
\text { FUNழূĀO DE PERDA }\end{array}$ & $\begin{array}{c}\text { 1' Parcela da Função de } \\
\text { Perda }\end{array}$ \\
\hline Menor-é-melhor & Perda $\uparrow$ & $\begin{array}{c}\text { Se } Y_{j} \leq T_{j} . D M=0 \\
\text { Se } Y_{j}>T_{j}, D M=\left(Y_{j}-T_{j}\right)^{2}\end{array}$ \\
\hline Maior-é-melhor & Perda $\uparrow$ & $\begin{array}{c}\text { Se } Y_{i}<T_{1} . D M=\left(Y_{1}-T_{i}\right)^{2} \\
S c Y_{i} \geq T_{1} . D M=0\end{array}$ \\
\hline Nominal-é-melhor & $\frac{x}{r_{1}}$ & $D M=\left(Y_{j}-T_{j}\right)^{2}$ \\
\hline
\end{tabular}




\section{PRODUÇÃO}

\section{Referências Bibliográficas}

BOX, G. E P. \& DRAPER, N. Empirical Model Building and Response Surfaces. John Wiley and Sons, New York, 1987.

CATEN, C.S. e RIBEIRO, J.L. Custos da qualidade e da manufatura: Um estudo de caso na indústria química. Relatório Técnico 06/95, Programa de Pós Graduação em Engenharia de Produção/ UFRGS, Porto Alegre, 1995.

CATEN, C.S. e RIBEIRO, J.L. Otimização experimental da qualidade: Massas cerâmicas e processo de fundição. Relatório Técnico 05/95, Programa de Pós Graduação em Engenharia de Produção/ UFRGS, Porto Alegre, 1995b.

CORNELL, J.A. Experiments with mixtures: a review. Technometrics, v. 15 , n.3, p. $437-455,1973$.

CORNELL, J.A. Experiments with mixtures: designs, models, and the analysis of mixture data. John Wiley and Sons, New York, 1981.

HEINSMAN, J.A. \& MONTGOMERY, D.C. Optimization of a household product formulation using a mixture experiment. Quality Engineering, v. 7, n.3, p. 583-600, 1995.

MONTGOMERY, D.C. Design and analysis of experiments. John Wiley and Sons, 2nd ed., New York, 1984.
MONTGOMERY, D.C. \& PECK Introduction to linear regression analysis. 2nd ed. John Wiley and Sons, New York, 1991.

RIBEIRO, J. L. \& ELSAYED, E. A. A case study on process optimization using the gradient loss function. International Journal of Production Research, Vol. 33, No. 12, pp. 3233-3248, 1995.

Artigo recebido em abril/1996, avaliado em junho/1996 e aprovado para publicação em julho/1996 